\title{
SKP2 confers resistance of pancreatic cancer cells towards TRAIL-induced apoptosis
}

\author{
SUSANNE SCHÜLER, SANDRA DIERSCH, RAINER HAMACHER, \\ ROLAND M. SCHMID, DIETER SAUR and GÜNTER SCHNEIDER \\ II. Medizinische Klinik, Technische Universität München, Ismaninger Str. 22, D-81675 München, Germany
}

Received July 30, 2010; Accepted September 27, 2010

DOI: 10.3892/ijo_00000841

\begin{abstract}
Pancreatic ductal adenocarcinoma (PDAC) is characterized by a dismal prognosis and no effective conservative therapy exists. Although the F-box protein S-phase kinase associated protein 2 (SKP2) is highly expressed and regulates cell cycle progression in PDAC, alternative SKP2 functions in PDAC are unknown. Using RNA interference we now demonstrate that SKP2 confers resistance of a subset of PDAC cell lines towards the tumor necrosis factor-related apoptosis-inducing ligand (TRAIL), but not the topoisomerase II inhibitor etoposide. We observed accelerated cleavage of the BH3-only protein Bid and augmented downregulation of cFLIPL, XIAP and MCL1 upon treatment of SKP2-depleted MiaPaCa2 cells with TRAIL. Our data disclose a novel SKP2 function in PDAC cells and therefore define SKP2 as a molecular target.
\end{abstract}

\section{Introduction}

Despite increasing knowledge concerning the molecular and genetic alterations of pancreatic ductal adenocarcinoma (PDAC) (1-7), the 5-year survival rate remains $<5 \%$ and no significant improvement in patient overall survival was documented over the last decades (8). In addition, most clinical phase III studies have failed to establish novel and efficient therapies for this disease $(7,9)$. Therefore, there is a need to increase our understanding of therapeutic resistance at the molecular level in PDAC.

Therapeutic resistance in PDACs can be divided in cell intrinsic and extrinsic mechanisms (10). Recent work in genetically engineered murine PDAC models, clearly demonstrated that cell extrinsic resistance contributes to therapeutic failure in PDAC. Here, it was demonstrated that the very low blood vessel density combined with the characteristic desmoplasia of PDAC contributes to impaired drug delivery and therapeutic failure (11). In addition to extrinsic therapeutic

Correspondence to: Dr Günter Schneider, Technische Universität München, Klinikum rechts der Isar, II. Medizinische Klinik, Ismaninger Str. 22, D-81675 Munich, Germany

E-mail: guenter.schneider@lrz.tum.de

Key words: SKP2, pancreatic cancer, apoptosis, TRAIL resistance, cell intrinsic therapeutic resistance accounts for the treatment failure of PDAC. Since many therapeutics induce apoptosis, evasion of apoptosis is a main mechanism contributing to cell intrinsic therapeutic resistance and the insufficiency of current therapies (12). Therefore, restoring the sensitivity of PDAC cells towards cell death inducers is a therapeutic goal $(13,14)$.

The oncogene S-phase kinase associated protein 2 (SKP2) is a F-box protein that functions as a receptor component of the SCF (Skp1-Cullin-F-box protein) ubiquitin ligase complex $(15,16)$. SKP2 is overexpressed in most human cancers and mediates oncogenic function by ubiquitination and degradation of proteins including the cyclin-dependent kinase inhibitors $\mathrm{p} 27^{\mathrm{Kip} 1}, \mathrm{p} 21^{\mathrm{Cip} 1}, \mathrm{p} 57^{\mathrm{Kip} 2}$ or the retinoblastoma-like 2 (p130) protein $(15,17)$. In PDAC cells, the SKP2 gene is activated by the phosphatidylinositol 3-kinase (PI3K)- and NF- $\mathrm{KB}$ signaling pathways $(18,19)$. Furthermore, high levels of SKP2 expression were observed in about one third of pancreatic cancer specimens and was shown to be an independent predictor of worse patient outcome (20). In this study we show that SKP2, in addition to the regulation of cell cycle progression, mediates resistance of PDAC cells towards tumor necrosis factor-related apoptosis-inducing ligand (TRAIL)induced apoptosis.

\section{Materials and methods}

Cell culture, siRNA transfection and reagents. The pancreatic cancer cell lines MiaPaCa2, Panc1, DanG and PatuII were cultivated as recently described $(18,19,21,22)$. TRAIL and etoposide were purchased from EMD (EMD Biosciences, San Diego, CA, USA). Untreated controls received vehicle alone. Double-stranded siRNAs were transfected at a final concentration of $50 \mathrm{nM}$ as described $(21,22)$. siRNAs were purchased from Eurofins, Ebersberg, Germany. Target sequences of the used siRNAs were: control siRNA 5'-CAG TCGCGTTTGCGACTGGdtdt-3', SKP2-1 siRNA 5'-GGGA GTGACAAAGACTTTGdtdt-3 ', SKP2-3 siRNA 5'-GCATG TACAGGTGGCTGTTdtdt-3'.

Statistical methods. All data were obtained from at least three independent experiments performed in triplicate, and the results are presented as mean and standard error of the mean (SEM). To demonstrate statistical significance a two-tailed Student's t-test was used. p-values are indicated, and an asterisk in the figures denotes $\mathrm{p}<0.05$. 
Quantitative reverse-transcriptase PCR. Total RNA was isolated from pancreatic carcinoma cell lines using the RNeasy kit (Qiagen, Hilden, Germany) following the manufacturer's instructions. Quantitative mRNA analyses were performed as previously described using real-time PCR analysis (TaqMan, PE Applied Biosystems, Norwalk, CT) $(21,22)$. Expression levels were normalized using cyclophilin. Primer sequences are available upon request.

Total cell lysates and Western blot analyses. Whole cell lysates were prepared and Western blot analyses were done as recently described $(18,19,21,22)$. The following antibodies were used: XIAP (R\&D Systems, Minneapolis, MN, USA); Bid, bcl $_{\mathrm{XL}}$ (Cell Signaling Technology Inc., Danvers, MA, USA); p27 ${ }^{\text {Kip1 }}$ (Santa Cruz Biotechnology, Santa Cruz, CA, USA); MCL1 (Alexis Biochemicals, San Diego, CA, USA); SKP2 (Invitrogen, Frankfurt, Germany); cFLIP, cleaved PARP (BD Biosciences, Heidelberg, Germany); $ß$-actin (SigmaAldrich, Munich, Germany). One representative Western blotting out of at least three independent experiments is shown. Western blot analyses were quantified using Odyssey Infrared Imaging System (LI-COR Biosciences, Bad Homburg, Germany).

Hoechst-stain, MTT- and Caspase-3/7-assay. Viability of the cells was measured using MTT-assays performed according to the manufacturer's protocol (Roche Applied Science, Mannheim, Germany). Caspase-3/7 activity was determined using Promega's Caspase-Glo-3/7 assay according to the manufacturer's instructions (Promega, Madison, WI, USA). Chromatin was stained with Hoechst $33342(4 \mu \mathrm{M})$ and typical apoptotic morphological changes were visualized using fluorescence microscopy as described (23). The apoptotic fractions of 300 cells were counted per individual experiment.

Cell cycle analysis. BrdU incorporation was measured using the colorimetric BrdU-Assay according to the manufacturer's instructions (Roche Applied Science). For cell cycle analysis, cells were washed twice in PBS and redissolved in propidium iodide (PI) staining buffer containing $0.1 \%$ sodium citrate, $0.1 \%$ Triton X-100, and $50 \mu \mathrm{g} / \mathrm{ml}$ PI. After $1 \mathrm{~h}$ of incubation at $4^{\circ} \mathrm{C}$, flow cytometry was performed using a BD Biosciences FACScan. The distribution of cells in different cell cycle stages $(\mathrm{G} 1 / \mathrm{S} / \mathrm{G} 2+\mathrm{M})$ was determined according to their DNA content as described $(18,19)$.

\section{Results}

SKP2 controls G1-phase progression and resistance of MiaPaCa2 cells towards TRAIL. Recently we have demonstrated that signaling pathways activating SKP2 transcription are linked to an accelerated G1- to S-phase progression $(18,19,24)$. To prove the contribution of SKP2 towards the cell cycle control in the model investigated, we used RNA interference (RNAi). Forty-eight hours after the transfection of a SKP2-specific siRNA, SKP2 protein (Fig. 1A) and mRNA levels (Fig. 1B) were distinctly decreased compared to control siRNA transfected MiaPaCa2 cells. Consistent with an important function of SKP2 for cell cycle regulation, we observed increased expression levels of the SKP2 target
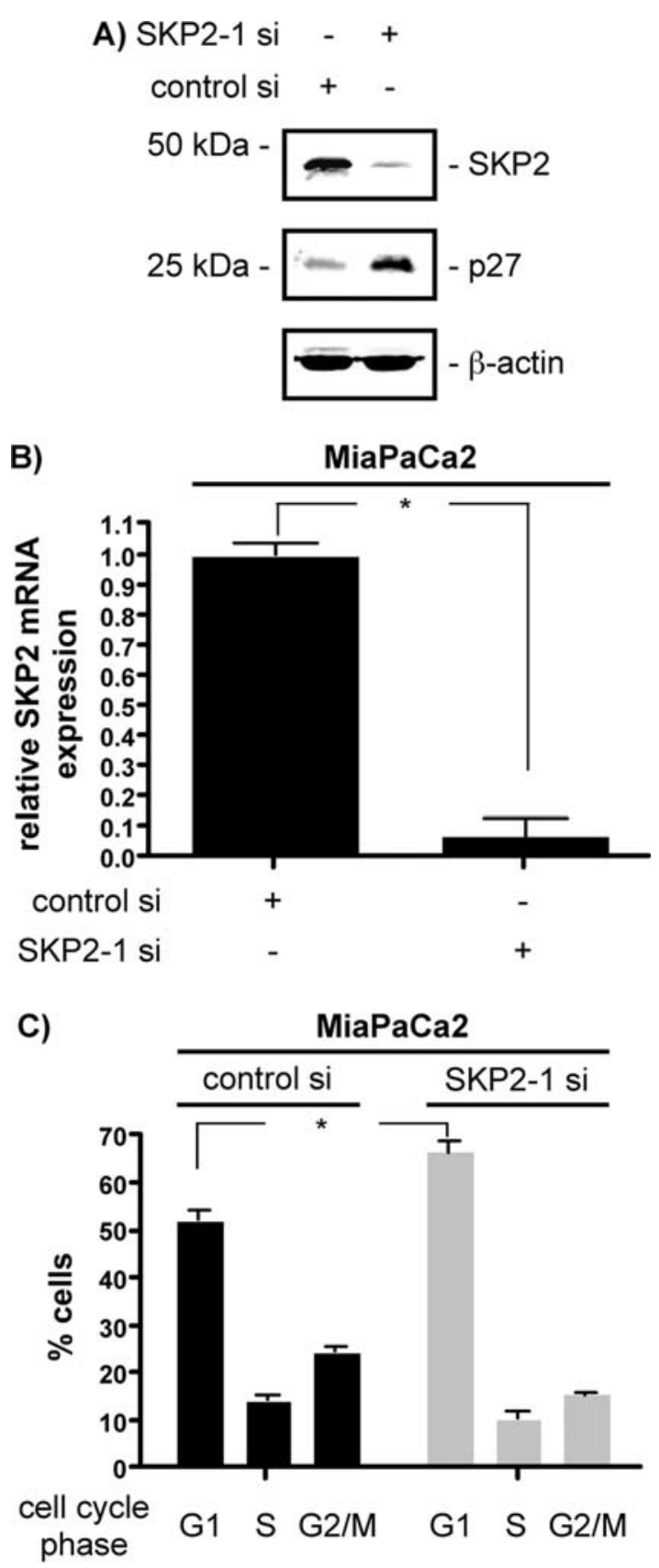

Figure 1. SKP2 knockdown induces a G1-phase arrest of the cell cycle in MiaPaCa2 cells. (A) Western blot analysis of SKP2 and p27 Kip1 $48 \mathrm{~h}$ after the transfection of MiaPaCa2 cells with a control siRNA or a SKP2-specific (SKP2-1) siRNA. ß-actin controls equal protein loading. (B) Quantitative SKP2 mRNA expression analysis in MiaPaCa2 cells after the transfection of a control or a SKP2-specific (SKP2-1) siRNA. Total RNA was prepared $48 \mathrm{~h}$ post-transfection. SKP2 mRNA levels were quantified using real-time PCR analysis and normalized to cyclophilin expression levels (Student's t-test: ${ }^{*} \mathrm{p}<0.05$ versus controls). (C) MiaPaCa2 cells were transfected with the indicated siRNAs. Forty-eight hours post-transfection, cells were stained with PI and cell cycle distribution was analyzed by FACS. The fraction of the cells in G1, S and G2/M phases is indicated (Student's t-test: ${ }^{*} \mathrm{p}<0.05$ versus controls).

p27 Kip1 (Fig. 1A) and a consecutive accumulation of the cells in the G1-phase of the cell cycle (Fig. 1C). Since the G1-phase of the cell cycle of PDAC cells is especially sensitive for TRAIL-induced apoptosis (25), we treated SKP2-depleted $\mathrm{MiaPaCa} 2$ cells with TRAIL and investigated viability and 
A)

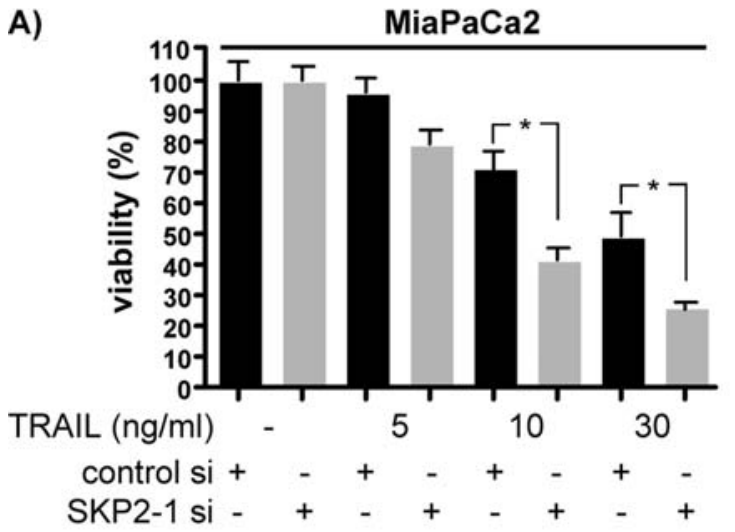

C)

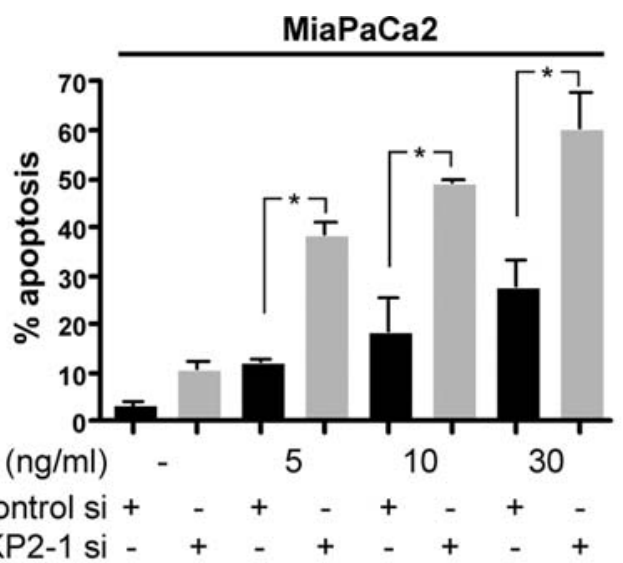

B)

SKP2

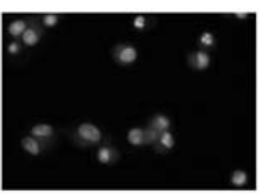

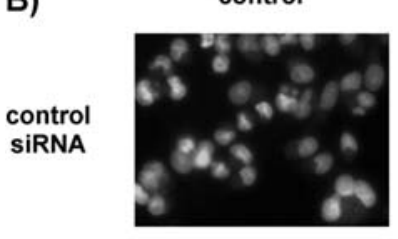

TRAIL
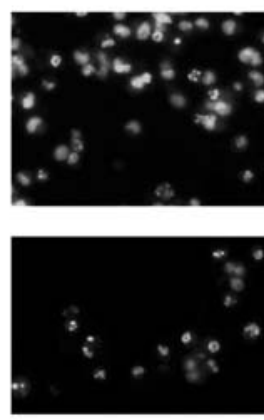

MiaPaCa2

E)

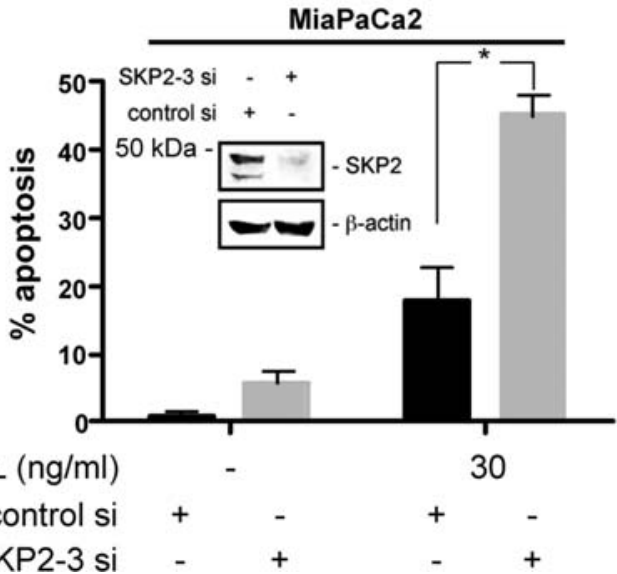

D)

$\mathrm{MiaPaCa} 2$

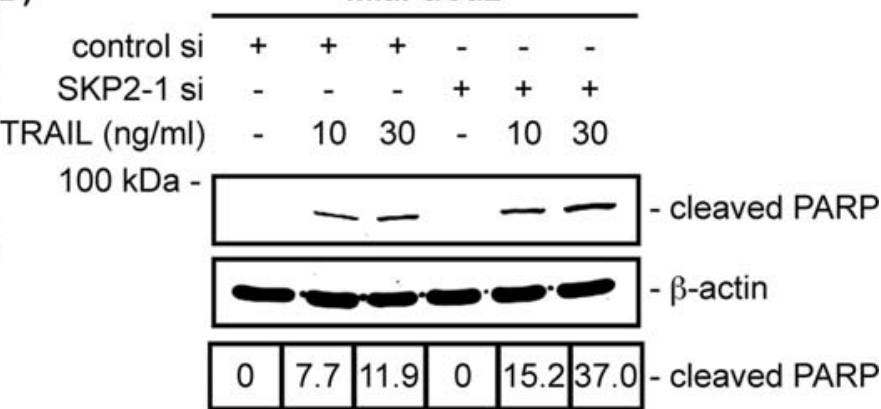

F)

$\mathrm{MiaPaCa} 2$

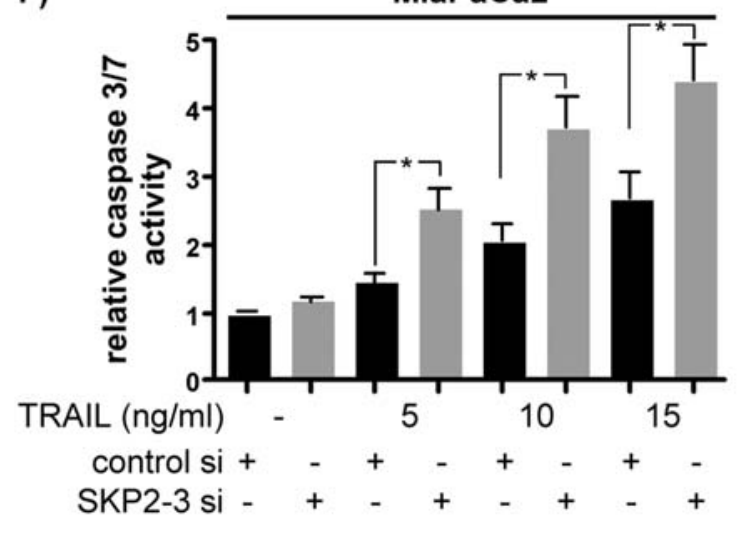

Figure 2. SKP2 controls TRAIL sensitivity of MiaPaCa2 cells. (A) MiaPaCa2 cells were transfected with a control siRNA or an SKP2-specific (SKP2-1) siRNA. Forty-eight hours after the transfection the cells were treated with increasing doses of TRAIL as indicated for additional $24 \mathrm{~h}$ or left as an untreated control. Viability was determined using MTT assays (Student's t-test: ${ }^{*} \mathrm{p}<0.05$ versus controls). (B) MiaPaCa2 cells were transfected with a control siRNA or an SKP2-specific (SKP2-1) siRNA. Forty-eight hours after the transfection the cells were treated with $30 \mathrm{ng} / \mathrm{ml}$ TRAIL for additional $24 \mathrm{~h}$ or left as an untreated control. Photomicrographs (original magnification x40) of MiaPaCa2 cells after the staining of the nuclei with Hoechst reveal apoptotic changes. (C) MiaPaCa2 cells were transfected with a control siRNA or an SKP2-specific (SKP2-1) siRNA. Forty-eight hours after transfection the cells were treated with increasing doses of TRAIL as indicated for additional $24 \mathrm{~h}$ or left as an untreated control. Apoptotic cells were quantified by fluorescence microscopy after Hoechst staining (Student's t-test: " p<0.05 versus controls). (D) MiaPaCa2 cells were transfected with a control siRNA or an SKP2-specific (SKP2-1) siRNA. Fortyeight hours after transfection the cells were treated with increasing doses of TRAIL as indicated for additional $24 \mathrm{~h}$ or left as an untreated control. Western blot analysis of PARP cleavage. B-actin controls equal protein loading. Cleaved PARP was quantified using the Odyssey near-infrared fluorescence scanner ensuring measurements in the linear range. Relative expression of cleaved PARP is indicated. (E) Western blot analysis of SKP2 $48 \mathrm{~h}$ after the transfection of MiaPaCa2 cells with a control siRNA or an SKP2-specific (SKP2-3) siRNA. B-actin controls equal protein loading. MiaPaCa2 cells were transfected with a control siRNA or an SKP2-specific (SKP2-3) siRNA. Forty-eight hours after transfection the cells were treated with TRAIL as indicated for additional 24 h or left as an untreated control. Apoptotic cells were quantified by fluorescence microscopy after Hoechst staining (Student's t-test: ${ }^{*} \mathrm{p}<0.05$ versus controls). (F) MiaPaCa2 cells were transfected with a control siRNA or an SKP2-specific (SKP2-3) siRNA. Forty-eight hours after transfection the cells were treated with TRAIL as indicated for additional $2 \mathrm{~h}$ or left as an untreated control. Caspase activity was measured using caspase-3/7 activity assays (Student's t-test: ${ }^{*}$ p $<0.05$ versus controls).

cell death. As shown in Fig. 2A, SKP2-depleted cells revealed an increased TRAIL-induced loss of viability, which was due to a distinctly increased apoptotic fraction (Fig. 2B and C).
SKP2-depleted MiaPaCa2 cells show an augmented caspase activation as measured indirectly by the cleavage of the caspase-substrate poly-(ADP-ribose) polymerase 1 (PARP) 

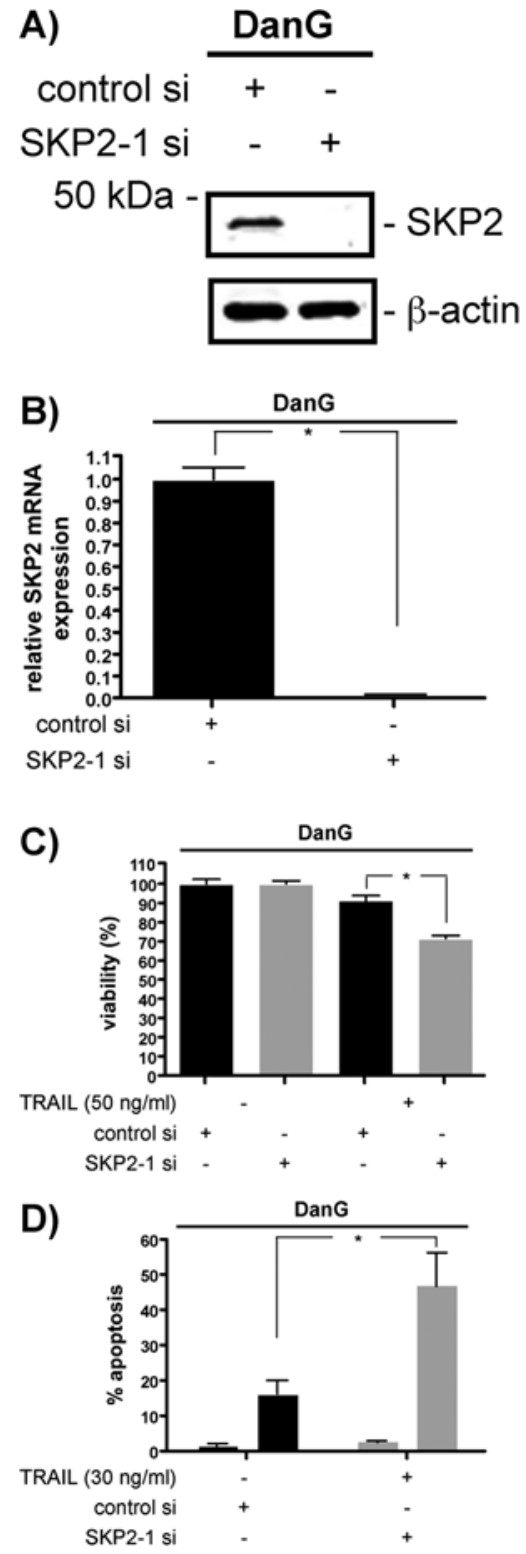
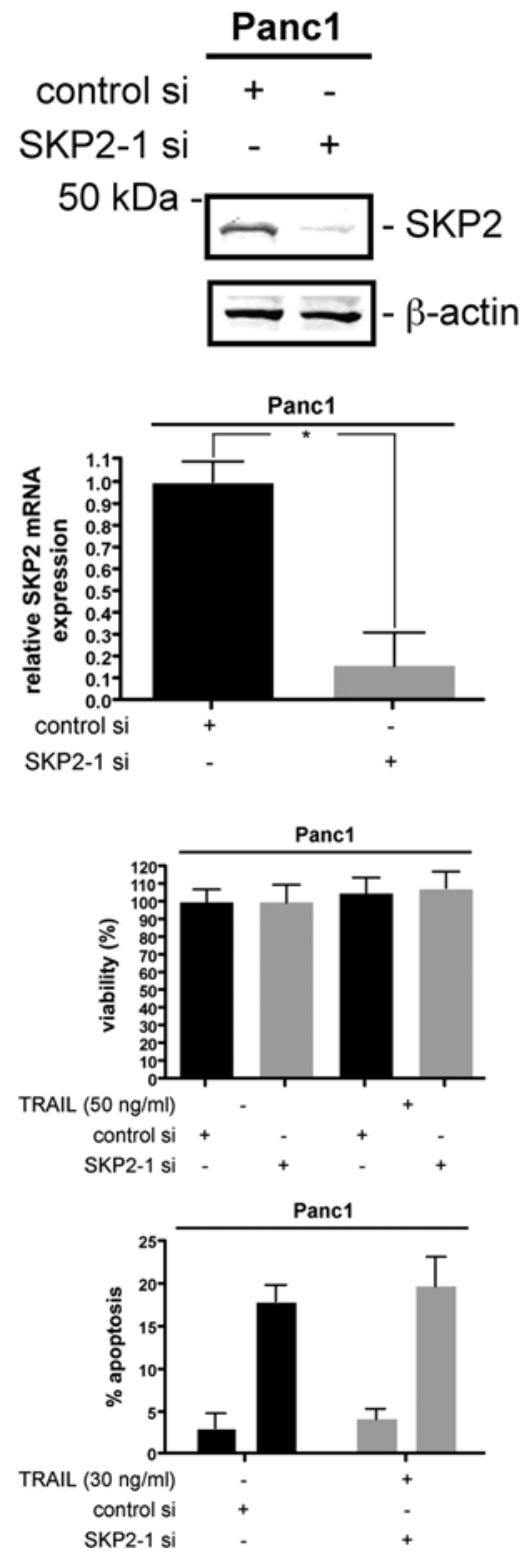
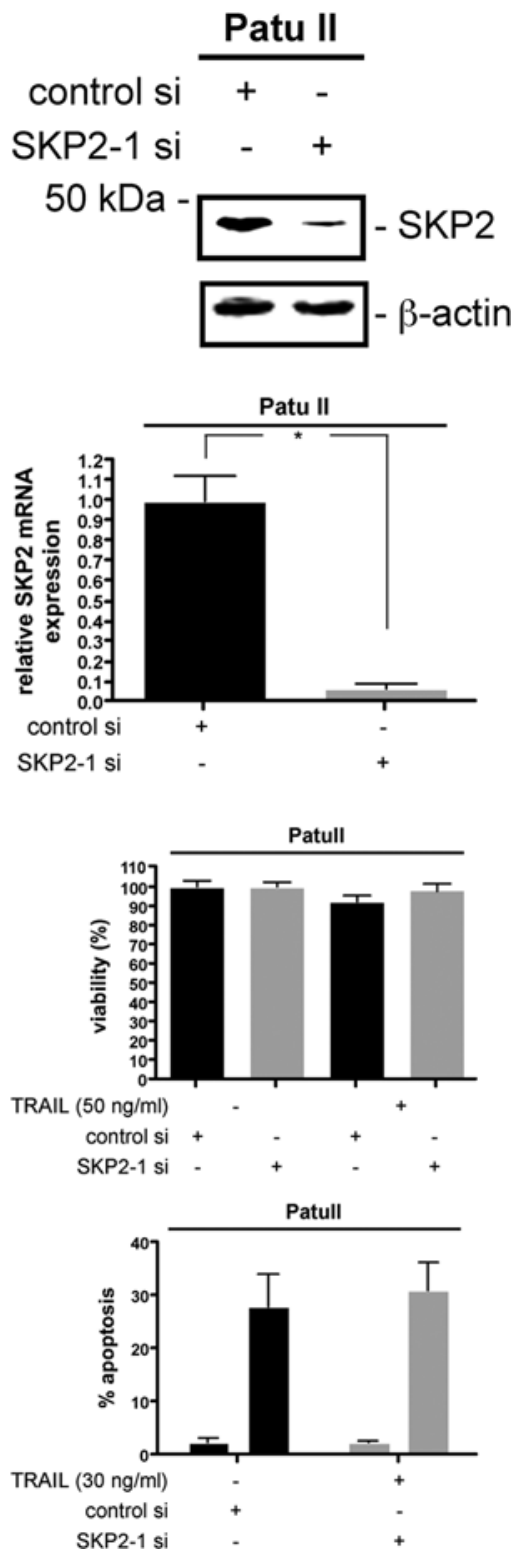

Figure 3. SKP2 and TRAIL sensitivity of DanG, Panc1 and PatuII cells. (A) Western blot analysis of SKP2 48 h after the transfection of DanG, Panc1 and PatuII cells with a control siRNA or an SKP2-specific (SKP2-1) siRNA. B-actin controls equal protein loading. (B) Quantitative SKP2 mRNA expression analysis in DanG, Panc1 and PatuII cells after the transfection of a control or an SKP2-specific (SKP2-1) siRNAs. Total RNA was prepared 48 h posttransfection. SKP2 mRNA levels were quantified using real-time PCR analysis and normalized to cyclophilin expression levels (Student's t-test: * $<0.05$ versus controls). (C) DanG, Panc1 and PatuII cells were transfected with a control siRNA or an SKP2-specific (SKP2-1) siRNA. 48 h after the transfection the cells were treated with $50 \mathrm{ng} / \mathrm{ml}$ TRAIL as indicated for additional $24 \mathrm{~h}$ or left as an untreated control. Viability was determined using MTT assays (Student's t-test: ${ }^{*}$ p $<0.05$ versus controls). (D) DanG, Panc1 and PatuII cells were transfected with a control siRNA or an SKP2-specific (SKP2-1) siRNA. Forty-eight hours after transfection the cells were treated with TRAIL as indicated for additional $24 \mathrm{~h}$ or left as an untreated control. Apoptotic cells were quantified by fluorescence microscopy after Hoechst staining (Student's t-test: *p<0.05 versus controls).

(Fig. 2D). To validate these results we used a second SKP2specific siRNA. Again, the SKP2 knockdown (Fig. 2E) lead to a prominent increase of the TRAIL-induced apoptotic fraction (Fig. 2E) and TRAIL-induced caspase activation (Fig. 2F). Together, these data suggest that SKP2 increases the sensitivity of $\mathrm{MiaPaCa} 2$ cells for TRAIL-induced apoptosis.

Control of TRAIL-sensitivity by SKP2 is cell-type specific. To determine whether SKP2 is generally involved in the regulation of sensitivity for TRAIL-induced apoptosis, we transfected the PDAC cell lines DanG, Panc1, and PatuII with the SKP2specific siRNA. The knockdown was verified $48 \mathrm{~h}$ after the transfection of the SKP2-specific siRNA at the level of SKP2 protein (Fig. 3A) and mRNA (Fig. 3B). Although depletion of SKP2 significantly increased TRAIL-induced loss of viability (Fig. 3C) and distinctly increased TRAIL-induced apoptotic fraction in DanG cells (Fig. 3D), SKP2 depletion did not change TRAIL sensitivity of Panc1 and PatuII cells (Fig. 3C and D). These data argue that SKP2-dependent regulation of TRAIL sensitivity is cell-type specific, reflecting tumor heterogeneity. 
A)

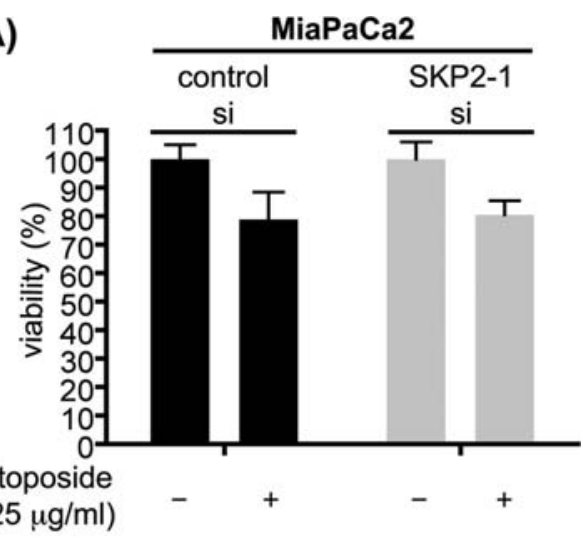

B)

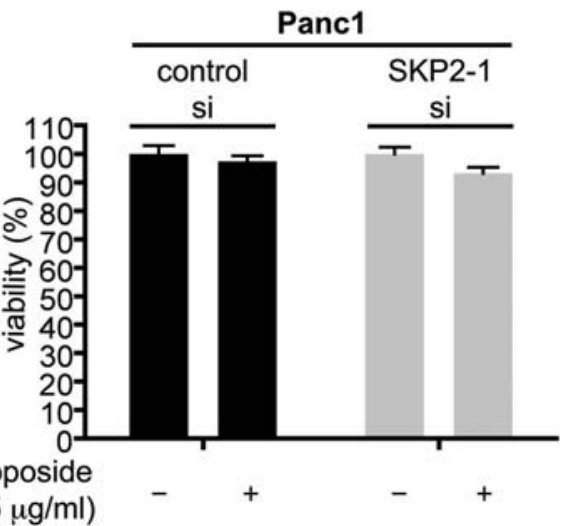

Figure 4. SKP2 and etoposide sensitivity of MiaPaCa2 and Panc1 cells (A) MiaPaCa2 and (B) Panc1 cells were transfected with a control siRNA or an SKP2-specific (SKP2-1) siRNA. Forty-eight hours after the transfection the cells were treated with etoposide as indicated for additional $24 \mathrm{~h}$ or left as an untreated control. Viability was determined using MTT assays.

SKP2 is not involved in regulation of topoisomerase II inhibitor sensitivity of PDAC cells. The use of DNA-damaging agents is a current strategy to treat solid tumors. Since recent work demonstrated that SKP2 confers resistance towards DNAdamage induced apoptosis $(26,27)$, we tested the sensitivity of SKP2-depleted PDAC cells towards the topoisomerase II inhibitor etoposide. As shown in Fig. 4, sensitivity of SKP2 depleted MiaPaCa2 (Fig. 4A) and Panc1 cells (Fig. 4B) was not changed compared to control siRNA transfected cells, suggesting that SKP2 is not linked to the regulation of topoisomerase II inhibitor sensitivity in PDAC cells.

TRAIL treatment leads to an accelerated cleavage of Bid and depletion of cFLIPL, XIAP and MCL1 in SKP2 depleted MiaPaCa2 cells. Sensitivity of PDAC cells towards TRAIL is controlled at the level of the death inducing signaling complex (DISC), at the level of the inhibitors of apoptosis proteins (IAPs), and, since PDAC cells are type II cells depending on the activation and cleavage of the $\mathrm{BH} 3$-only protein $\mathrm{Bid}$, at the level of the mitochondrium $(13,14)$. Consistent with increased sensitivity towards TRAIL-induced apoptosis, we observed accelerated cleavage of Bid in SKP2depleted MiaPaCa2 cells over time (Fig. 5). Twenty-four hours after TRAIL treatment we detected a slightly pronounced depletion of XIAP and $\mathrm{CFLIP}_{\mathrm{L}}$ in SKP2 siRNA transfected MiaPaCa2 cells. Furthermore, we observed already $6 \mathrm{~h}$ after

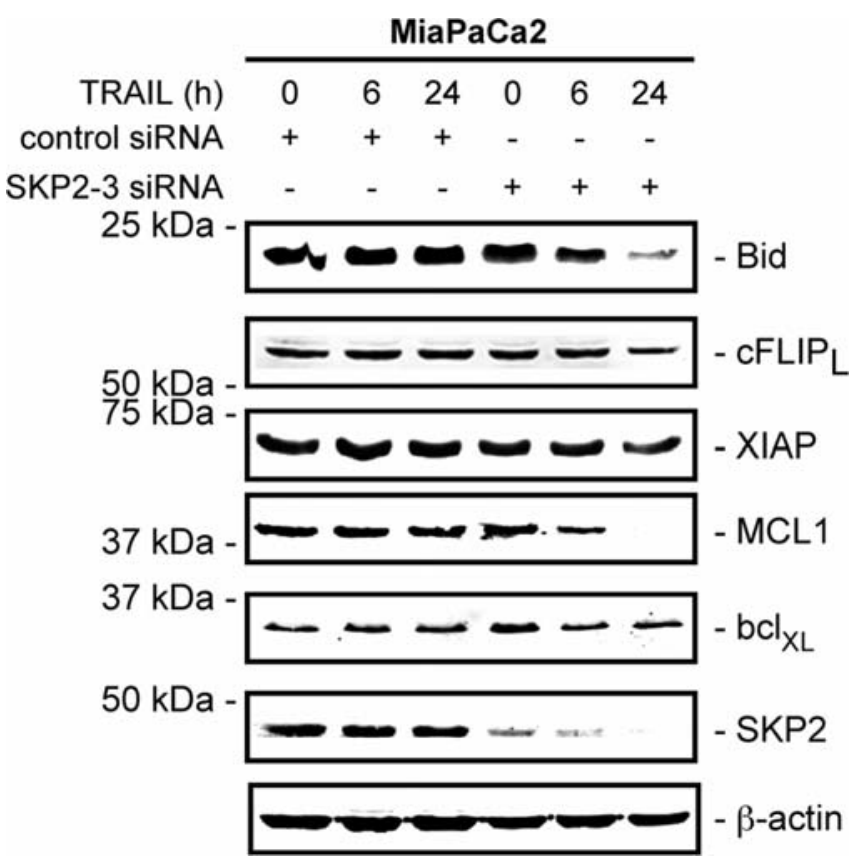

Figure 5. SKP2 and regulation of TRAIL resistance proteins in MiaPaCa2 cells. Forty-eight hours after the transfection of MiaPaCa2 cells with a control siRNA or an SKP2-specific siRNA cells were treated with TRAIL over time as indicated. Western blot analysis of Bid, cFLIP $_{\mathrm{L}}$, XIAP, MCL1, $\mathrm{bcl}_{\mathrm{XL}}$ and SKP2. ß-actin controls equal protein loading.

the TRAIL treatment a downregulation of the anti-apoptotic BCL2 family member MCL1 in SKP2 siRNA transfected MiaPaCa2 cells (Fig. 5). Whereas the basal expression of $\mathrm{bcl}_{\mathrm{XL}}$ was slightly increased in SKP2-depleted MiaPaCa2 cells (Fig. 5), basal expression of cIAP1, cIAP2 and survivin was not changed in SKP2-depleted MiaPaCa2 cells (data not shown).

\section{Discussion}

TRAIL agonistic therapeutic approaches are currently investigated in various clinical trials (28-31). In PDAC, high TRAIL expression was correlated with an increased apoptotic index in the human pancreas arguing that a TRAIL-based therapy might be a feasible strategy for the treatment of PDAC $(32,33)$. Nevertheless, primary human tumor cells often resist TRAIL-induced apoptosis (34) and PDAC cells reveal a high half-maximal inhibitor concentration $\left(\mathrm{IC}_{50}\right)$ for TRAIL despite they express the TRAIL receptors DR4 (TRAIL-R1) and DR5 (TRAIL-R2) as well as relevant mediators of the TRAIL receptor signaling pathway $(13,14,35)$. Therefore, defining molecular targets whose inhibition synergizes with TRAIL agonists is important for defining new therapies. Several proteins, conferring TRAIL resistance to PDAC cells, including histone deacetylase 2 (HDAC2) (21), STAT3 (36), CUX1 (37), cFLIP (38-40), XIAP (41-45), MCL1 $(36,46)$, bcl $_{\mathrm{XL}}(36,47,48)$ or survivin $(25)$ were described. In addition our current work now demonstrates that SKP2 can confer TRAIL resistance to PDAC cells.

The most prominent function of SKP2 is to drive cell cycle progression, mainly by inducing ubiquitination and degradation 
of $\mathrm{p} 27^{\mathrm{Kip} 1}(15-17)$. However, recent evidence revealed that SKP2 can also regulate cancer-relevant processes like senescence (49), anoikis (50) or apoptosis (26,27,51-56). To our knowledge, we provide for the first time evidence that SKP2, highly expressed in pancreatic cancer (20), confers resistance towards extrinsic induction of apoptosis. Although we detected accelerated Bid cleavage and slightly pronounced depletion of cFLIP $_{\mathrm{L}}$ and XIAP, the molecular mechanism by which SKP2 controls TRAIL sensitivity remains unclear at the moment and the described changes can thus occur secondary to increased TRAIL-induced apoptosis in SKP2-depleted cells. In contrast to this late occurring events, the expression of MCL1, a pro-survival BCL2 family member, was already decreased $6 \mathrm{~h}$ after treatment with TRAIL in SKP2 depleted cells. Although MCL1 can protect PDAC cells from TRAIL(36), TNF $\alpha$ - and chemotherapy-induced apoptosis (unpublished data), the regulation of this short-lived protein in PDAC cells is complex and regulation of MCL1 occurs at the level of transcription, translation, and degradation (57). How, directly or indirectly, SKP2 is involved in the regulation of MCL1 in the model system investigated is unclear and awaits further investigation.

SKP2 regulates the response of cancer cells towards DNA-damaging agents $(26,27,53)$. At the molecular level, SKP2 was shown to suppress p53-dependent apoptosis by forming a complex with the histone acetyltransferase p300. Thereby, SKP2 is antagonizing the p53/p300 interaction, needed to activate pro-apoptotic programs (26). We did not observe changes of PDAC cells sensitivity towards etoposide-induced apoptosis in SKP2-depleted cells. The fact that MiaPaCa2 ( $\left.53^{\mathrm{R} 248 \mathrm{~W}}\right)$ as well as Panc1 ( $\left.\mathrm{p} 53^{\mathrm{R} 273 \mathrm{H}}\right)$ cells harbor p53 mutations might explain this discrepancy.

Collectively, additional studies are needed to decipher the regulation of TRAIL sensitivity by SKP2 in molecular detail. Nevertheless, our findings provide clear evidence that SKP2 can contribute to the resistance of cancer cells towards extrinsic induction of apoptosis.

\section{Acknowledgements}

We thank Birgit Kohnke-Ertel and Kerstin Hoffmann for excellent technical support. This work was funded by Fritz Thyssen Stiftung (Az. 10.08.2.170 to G.S.) and Else KrönerFresenius Stiftung (A130/07 to G.S.).

\section{References}

1. Schneider G, Siveke JT, Eckel F and Schmid RM: Pancreatic cancer: basic and clinical aspects. Gastroenterology 128: $1606-1625,2005$

2. Koorstra JB, Hustinx SR, Offerhaus GJ and Maitra A: Pancreatic carcinogenesis. Pancreatology 8: 110-125, 2008.

3. Mihaljevic AL, Michalski CW, Friess $\mathrm{H}$ and Kleeff J: Molecular mechanism of pancreatic cancer-understanding proliferation, invasion, and metastasis. Langenbecks Arch Surg 395: 295-308, 2010.

4. Wescott MP and Rustgi AK: Pancreatic cancer: translating lessons from mouse models and hereditary syndromes. Cancer Prev Res 1: 503-506, 2008.

5. Buchholz M and Gress TM: Molecular changes in pancreatic cancer. Expert Rev Anticancer Ther 9: 1487-1497, 2009.

6. Wong $\mathrm{HH}$ and Lemoine NR: Pancreatic cancer: molecular pathogenesis and new therapeutic targets. Nat Rev Gastroenterol Hepatol 6: 412-422, 2009.
7. Hidalgo M: Pancreatic cancer. N Engl J Med 362: 1605-1617, 2010.

8. Mitry E, Rachet B, Quinn MJ, Cooper N and Coleman MP: Survival from cancer of the pancreas in England and Wales up to 2001. Br J Cancer 99 (Suppl. 1): S21-S23, 2008.

9. Eckel F, Schneider G and Schmid RM: Pancreatic cancer: a review of recent advances. Expert Opin Investig Drugs 15: 1395-1410, 2006.

10. Schneider G, Krämer OH, Fritsche P, Schuler S, Schmid RM and Saur D: Targeting histone deacetylases in pancreatic ductal adenocarcinoma. J Cell Mol Med 14: 1255-1263, 2010.

11. Olive KP, Jacobetz MA, Davidson CJ, et al: Inhibition of Hedgehog signaling enhances delivery of chemotherapy in a mouse model of pancreatic cancer. Science 324: 1457-1461, 2009.

12. Fulda $\mathrm{S}$ and Debatin KM: Extrinsic versus intrinsic apoptosis pathways in anticancer chemotherapy. Oncogene 25: 4798-4811, 2006.

13. Hamacher R, Schmid RM, Saur D and Schneider G: Apoptotic pathways in pancreatic ductal adenocarcinoma. Mol Cancer 7: 64, 2008.

14. Fulda S: Apoptosis pathways and their therapeutic exploitation in pancreatic cancer. J Cell Mol Med 13: 1221-1227, 2009.

15. Frescas D and Pagano M: Deregulated proteolysis by the F-box proteins SKP2 and beta-TrCP: tipping the scales of cancer. Nat Rev Cancer 8: 438-449, 2008.

16. Nakayama KI and Nakayama K: Regulation of the cell cycle by SCF-type ubiquitin ligases. Semin Cell Dev Biol 16: 323-333, 2005.

17. Hershko DD: Oncogenic properties and prognostic implications of the ubiquitin ligase Skp2 in cancer. Cancer 112: 1415-1424, 2008.

18. Reichert M, Saur D, Hamacher R, Schmid RM and Schneider G: Phosphoinositide-3-kinase signaling controls S-phase kinaseassociated protein 2 transcription via E2F1 in pancreatic ductal adenocarcinoma cells. Cancer Res 67: 4149-4156, 2007.

19. Schneider G, Saur D, Siveke JT, Fritsch R, Greten FR and Schmid RM: IKKalpha controls p52/RelB at the skp2 gene promoter to regulate G1- to S-phase progression. EMBO J 25: 3801-3812, 2006

20. Einama T, Kagata Y, Tsuda H, et al: High-level Skp2 expression in pancreatic ductal adenocarcinoma: correlation with the extent of lymph node metastasis, higher histological grade, and poorer patient outcome. Pancreas 32: 376-381, 2006.

21. Schüler S, Fritsche P, Diersch S, et al: HDAC2 attenuates TRAIL-induced apoptosis of pancreatic cancer cells. Mol Cancer 9: 80, 2010.

22. Fritsche P, Seidler B, Schüler S, et al: HDAC2 mediates therapeutic resistance of pancreatic cancer cells via the BH3-only protein NOXA. Gut 58: 1399-1409, 2009.

23. Hamacher R, Saur D, Fritsch R, Reichert M, Schmid RM and Schneider G: Casein kinase II inhibition induces apoptosis in pancreatic cancer cells. Oncol Rep 18: 695-701, 2007.

24. Schneider G, Reichert M, Saur D, Hamacher R, Fritsch R and Schmid RM: HDAC3 is linked to cell cycle machinery in $\mathrm{MiaPaCa} 2$ cells by regulating transcription of skp2. Cell Prolif 40: 522-531, 2007.

25. Retzer-Lidl M, Schmid RM and Schneider G: Inhibition of CDK4 impairs proliferation of pancreatic cancer cells and sensitizes towards TRAIL-induced apoptosis via downregulation of survivin. Int J Cancer 121: 66-75, 2007.

26. Kitagawa M, Lee SH and McCormick F: Skp2 suppresses p53-dependent apoptosis by inhibiting p300. Mol Cell 29: 217-231, 2008.

27. Sun L, Cai L, Yu Y, et al: Knockdown of S-phase kinaseassociated protein-2 expression in MCF-7 inhibits cell growth and enhances the cytotoxic effects of epirubicin. Acta Biochim Biophys Sin (Shanghai) 39: 999-1007, 2007.

28. Johnstone RW, Frew AJ and Smyth MJ: The TRAIL apoptotic pathway in cancer onset, progression and therapy. Nat Rev Cancer 8: 782-798, 2008.

29. Papenfuss K, Cordier SM and Walczak H: Death receptors as targets for anti-cancer therapy. J Cell Mol Med 12: 2566-2585, 2008.

30. Newsom-Davis T, Prieske S and Walczak H: Is TRAIL the holy grail of cancer therapy? Apoptosis 14: 607-623, 2009.

31. Russo M, Mupo A, Spagnuolo C and Russo GL: Exploring death receptor pathways as selective targets in cancer therapy. Biochem Pharmacol 80: 674-682, 2010. 
32. Sanlioglu AD, Dirice E, Elpek O, et al: High levels of endogenous tumor necrosis factor-related apoptosis-inducing ligand expression correlate with increased cell death in human pancreas. Pancreas 36: 385-393, 2008.

33. Sanlioglu AD, Dirice E, Elpek O, et al: High TRAIL death receptor 4 and decoy receptor 2 expression correlates with significant cell death in pancreatic ductal adenocarcinoma patients. Pancreas 38: 154-160, 2009.

34. Koschny R, Walczak H and Ganten TM: The promise of TRAIL-potential and risks of a novel anticancer therapy. J Mol Med 85: 923-935, 2007.

35. Ozawa F, Friess H, Kleeff J, et al: Effects and expression of TRAIL and its apoptosis-promoting receptors in human pancreatic cancer. Cancer Lett 163: 71-81, 2001.

36. Huang S and Sinicrope FA: Sorafenib inhibits STAT3 activation to enhance TRAIL-mediated apoptosis in human pancreatic cancer cells. Mol Cancer Ther 9: 742-750, 2010.

37. Ripka S, Neesse A, Riedel J, et al: CUX1: target of Akt signalling and mediator of resistance to apoptosis in pancreatic cancer. Gut 59: 1101-1110, 2010.

38. Kauh J, Fan S, Xia M, et al: c-FLIP degradation mediates sensitization of pancreatic cancer cells to TRAIL-induced apoptosis by the histone deacetylase inhibitor LBH589. PLoS One 5: e10376, 2010.

39. Wang P, Zhang J, Bellail A, et al: Inhibition of RIP and c-FLIP enhances TRAIL-induced apoptosis in pancreatic cancer cells. Cell Signal 19: 2237-2246, 2007.

40. Murtaza I, Saleem M, Adhami VM, Hafeez BB and Mukhtar H: Suppression of cFLIP by lupeol, a dietary triterpene, is sufficient to overcome resistance to TRAIL-mediated apoptosis in chemoresistant human pancreatic cancer cells. Cancer Res 69: 1156-1165, 2009.

41. Vogler M, Durr K, Jovanovic M, Debatin KM and Fulda S: Regulation of TRAIL-induced apoptosis by XIAP in pancreatic carcinoma cells. Oncogene 26: 248-257, 2007.

42. Vogler M, Walczak H, Stadel D, et al: Targeting XIAP bypasses Bcl-2-mediated resistance to TRAIL and cooperates with TRAIL to suppress pancreatic cancer growth in vitro and in vivo. Cancer Res 68: 7956-7965, 2008.

43. Vogler M, Walczak H, Stadel D, et al: Small molecule XIAP inhibitors enhance TRAIL-induced apoptosis and antitumor activity in preclinical models of pancreatic carcinoma. Cancer Res 69: 2425-2434, 2009.

44. Mori T, Doi R, Kida A, et al: Effect of the XIAP inhibitor Embelin on TRAIL-induced apoptosis of pancreatic cancer cells. J Surg Res 142: 281-286, 2007.
45. Dineen SP, Roland CL, Greer R, et al: Smac mimetic increases chemotherapy response and improves survival in mice with pancreatic cancer. Cancer Res 70: 2852-2861, 2010.

46. Huang S, Okumura K and Sinicrope FA: BH3 mimetic obatoclax enhances TRAIL-mediated apoptosis in human pancreatic cancer cells. Clin Cancer Res 15: 150-159, 2009.

47. Bai J, Sui J, Demirjian A, Vollmer CM Jr, Marasco W and Callery MP: Predominant Bcl-XL knockdown disables antiapoptotic mechanisms: tumor necrosis factor-related apoptosisinducing ligand-based triple chemotherapy overcomes chemoresistance in pancreatic cancer cells in vitro. Cancer Res 65: 2344-2352, 2005

48. Song JJ, An JY, Kwon YT and Lee YJ: Evidence for two modes of development of acquired tumor necrosis factor-related apoptosis-inducing ligand resistance. Involvement of Bcl-xL. J Biol Chem 282: 319-328, 2007.

49. Lin HK, Chen Z, Wang G, et al: Skp2 targeting suppresses tumorigenesis by Arf-p53-independent cellular senescence. Nature 464: 374-379, 2010.

50. Wang XC, Wu YP, Ye B, et al: Suppression of anoikis by SKP2 amplification and overexpression promotes metastasis of esophageal squamous cell carcinoma. Mol Cancer Res 7: 12-22, 2009.

51. Wang H, Bauzon F, Ji P, et al: Skp2 is required for survival of aberrantly proliferating Rb1-deficient cells and for tumorigenesis in $\mathrm{Rb}^{+/-}$mice. Nat Genet 42: 83-88, 2010.

52. Harada K, Supriatno, Kawashima Y, Itashiki Y, Yoshida H and Sato M: Down-regulation of S-phase kinase associated protein 2 (Skp2) induces apoptosis in oral cancer cells. Oral Oncol 41: 623-630, 2005.

53. Ishii T, Matsuse T, Masuda $M$ and Teramoto $S$ : The effects of S-phase kinase-associated protein 2 (SKP2) on cell cycle status, viability, and chemoresistance in A549 lung adenocarcinoma cells. Exp Lung Res 30: 687-703, 2004.

54. Yokoi S, Yasui K, Iizasa T, Takahashi T, Fujisawa T and Inazawa J: Down-regulation of SKP2 induces apoptosis in lungcancer cells. Cancer Sci 94: 344-349, 2003.

55. Lee SH and McCormick F: Downregulation of Skp2 and p27/Kip1 synergistically induces apoptosis in T98G glioblastoma cells. J Mol Med 83: 296-307, 2005.

56. Masuda TA, Inoue $\mathrm{H}$, Sonoda $\mathrm{H}$, et al: Clinical and biological significance of S-phase kinase-associated protein 2 (Skp2) gene expression in gastric carcinoma: modulation of malignant phenotype by Skp2 overexpression, possibly via p27 proteolysis. Cancer Res 62: 3819-3825, 2002.

57. Michels J, Johnson PW and Packham G: Mcl-1. Int J Biochem Cell Biol 37: 267-271, 2005 